\title{
Renal disease in relatives of Indo-Asian Type 2 diabetic patients with end-stage diabetic nephropathy
}

\author{
P. K. Chandie Shaw ${ }^{1}$, L. A. van Es ${ }^{2}$, L. C. Paul ${ }^{2}$, F. R. Rosendaal ${ }^{3}$, J. H. M. Souverijn ${ }^{4}$, J. P. Vandenbroucke ${ }^{3}$ \\ ${ }^{1}$ Department of Nephrology and Internal Medicine, Medical Center Haaglanden, The Hague, The Netherlands \\ 2 Department of Nephrology, Leiden University Medical Center, Leiden, The Netherlands \\ ${ }^{3}$ Department of Clinical Epidemiology, Leiden University Medical Center, Leiden, The Netherlands \\ ${ }^{4}$ Clinical Chemistry Laboratory, Leiden University Medical Center, Leiden, The Netherlands
}

\begin{abstract}
Aims/Hypothesis. Indo-Asian immigrants in The Hague, The Netherlands, have a nearly 40 -fold higher risk of end-stage diabetic nephropathy compared to the Caucasian population. To detect a genetic susceptibility for nephropathy within the Indo-Asian population, we assessed whether familial clustering of nephropathy occurs in families of Indo-Asian Type 2 diabetic patients. Methods. We compared nephropathy prevalence between two groups of first-degree relatives of IndoAsian patients with Type 2 diabetes; the first group (case relatives) consisted of 169 relatives of patients with end-stage diabetic nephropathy; the second group (control relatives) consisted of 161 relatives of diabetic patients who had no nephropathy. The case and control relatives were examined for diabetes, blood pressure, renal function, microalbuminuria and urine dipstick measurements.
\end{abstract}

Results. The mean age was 41 years and similar in the case and control relatives. Diabetes was distributed equally in both family groups. We did not find more nephropathy in first-degree relatives of Indo-Asian Type 2 diabetic patients with end-stage diabetic nephropathy in comparison with the controlrelatives.

Conclusion/interpretation. We could not detect a genetic susceptibility for diabetic nephropathy within the Indo-Asian population. The lack of familial clustering of renal disease in Indo-Asian diabetic patients points to a general genetic or environmental susceptibility for diabetic nephropathy in this population. [Diabetologia (2003) 46:618-624]

Keywords Albuminuria, genetics, diabetic nephropathy, ethnology, Indo-Asian, Asian, Indian, epidemiology, proteinuria, non insulin-dependent diabetes, DM Type 2.
Familial clustering of diabetic nephropathy was first described in Type 1 diabetic patients.[1] Later, clustering was also observed in Type 2 diabetic patients. A familial predisposition for diabetic nephropathy was observed in different ethnic groups like the American

Received: 9 August 2002 / Revised: 5 January 2003

Published online: 9 May 2003

C Springer-Verlag 2003

Corresponding author: Dr. P. K. Chandie Shaw, Department of Nephrology and Internal Medicine, Medical Center Haaglanden, P.O.Box 432, 2501 CK The Hague, The Netherlands

E-mail: P.Chandie@MCHaaglanden.nl

Abbreviations: BSA, Body square area; GP, general practitioner; GTT, Glucose tolerance test; NA, Not applicable; WHO, World Health Organization; 95\%-CI, 95\% confidence interval.
Pima Indians, Afro-Americans, Brazilian and Italian Type 2 diabetic patients.[2, 3, 4, 5, 6, 7]. These observations are consistent with the hypothesis of a genetic susceptibility in the pathogenesis of diabetic nephropathy.

Indo-Asian people from Surinam are originally descended from the Indian subcontinent, including India, Pakistan, and Bangladesh. In a recent study, IndoAsian patients were found to have a close to 40 -fold increased risk for end-stage diabetic nephropathy in comparison to native Caucasians [8]. Studies carried out in the United Kingdom showed comparable results $[9,10]$. This is higher than expected since the prevalence of Type 2 diabetes was only eight times higher in the Indo-Asian population, again in comparison with the Caucasian native population [11]. An expla- 
nation for this relatively higher incidence of diabetic nephropathy could be an additional genetic susceptibility to develop nephropathy within the Indo-Asian population. This might be detected by the presence of familial clustering of nephropathy in relatives of patients with diabetic nephropathy, but specific family studies for nephropathy are lacking in the Indo-Asian population. However, a small case-control family study in South India showed higher rates of proteinuria in siblings of Type 2 diabetic patients with nephropathy in comparison with age-, sex- and diabetic duration-matched siblings of control patients without diabetic nephropathy [12].

The aim of our study was to investigate whether familial clustering of nephropathy occurs in first degree relatives of Type 2 diabetic Indo-Asian patients with and without nephropathy. We tried to prevent selection bias by a population-based study design and the testing of all non-diabetic relatives, with an oral glucose tolerance test.

\section{Subjects and methods}

\section{Study design}

We evaluated the predisposition for nephropathy among IndoAsian first degree relatives of Type 2 diabetic patients with end-stage renal failure (case-relatives). They were compared with first-degree relatives of Indo-Asian Type 2 diabetic patients who had no clinical signs of diabetic nephropathy (control-relatives). The case and control relatives were invited for an assessment between September 1st, 1998 and December 31 st, 2000.

The study protocol was approved by the Institutional Medical Ethics Committee in accordance with the Declaration of Helsinki.

\section{Subjects}

Case-families. The case-index patients were recruited from the records of the dialysis units of three regional hospitals, which together represent the total dialysis capacity in the town of The Hague. Included were all patients first registered between 1990 and 1999 for dialysis because of end-stage renal failure attributed to Type 2 diabetes; patients were considered to have Type 2 diabetes if they used oral anti-diabetic medication prior to dialysis, or if their fasting C-peptide concentrations were indicative of Type 2 diabetes. Indo-Asian dialysis patients were initially identified by their surnames. If the patients were alive, we visited them in the dialysis unit and confirmed their ethnic origin. A standardized interview was taken with respect to diabetes and family history as well as demographic parameters. After informed consent, we contacted their first-degree relatives living in the Netherlands (parents, siblings and children). If the case-index patient had died, we contacted the relatives with the help of the general practitioner (GP) of the deceased patient.

Control families. Control-index patients with Type 2 diabetes were selected with the help of general practitioners of the included case-index patients. For each case-index patient includ- ed, one Indo-Asian control-index was chosen at random among the patients with Type 2 diabetes from the records of the GP; control-index patients were eligible if they were of the same sex as the case-index and had no microalbuminuria. To ensure random sampling, we went through all the records of that GP, and made a numbered list of all eligible Indo-Asian Type 2 patients. Subsequently, control-index patients were drawn from this list by use of a random number table. Another source of control-index patients were the spouses of the investigated relatives. If the spouse had Type 2 diabetes mellitus and no microalbuminuria, we invited the siblings and parents of the spouse for the investigation. The control-index patients were also invited through a letter for a visit at our outpatient's research unit. A few days later they were contacted by phone to obtain informed consent and to make an appointment at our research outpatient clinic.

Inclusion of the family relatives. All first-degree relatives (father, mother, siblings, and children) of the case and control index patients living in the Netherlands were invited as part of a family investigation for diabetes and renal disease. We invited the case and control relatives randomly throughout the investigation period. Relatives who were pregnant were invited later on, three months after they gave birth. Patients younger than 16 years were not included. We tried to avoid appointments during the menstrual period of women.

Procedures and measurements

The family relatives came during the morning hours, after fasting for at least $8 \mathrm{~h}$. Fasting venous blood samples were drawn for haemoglobin, creatinin and lipid profile. The relatives brought an early morning urine sample for quantitative measurements of albuminuria and dipstick urine analysis. They stayed in a quiet room and the blood pressure was measured three times after a 5-min rest in sitting position using an OMRON 705CP automatic oscillometric blood pressure device. The cuff was placed at the right upper arm. If the circumference of the arm exceeded $32 \mathrm{~cm}$, a large cuff was used. The weight and height were recorded while wearing underwear. Also the circumference of the waist and hip were measured. If the relatives did not use anti-diabetic medication, an OGTT was done with $75 \mathrm{~g}$ of glucose and the fasting glucose as well as 2-h glucose was measured. The renal function was estimated using the Cockcroft-Gault estimation [13]. We used a questionnaire to obtain data on general demographic variables (age, sex, educational level and marital status) family history of diabetes mellitus in first-degree relatives (age of onset, duration, treatment), hypertension, smoking and medication.

\section{Laboratory measurements}

Urinary albumin and protein were measured by immunoturbidimetric assay on a Hitachi 911, as was the HDL-cholesterol in serum. Glucose, creatinin, cholesterol and triglycerides were measured on a Hitachi-747 (Hitachi Tokyo, Japan). HbA $\mathrm{lc}_{\mathrm{c}}$ was measured using the HPLC method with a Variant analyser (Biorad, Hercules, Calif., USA). The Variance Coefficient was $1.5 \%$ at different levels. The reference values for $\mathrm{HbA}_{1 \mathrm{c}}$ are between 4.3 and $6.3 \%$. Urine dipstick investigation for leukocyturia and hematuria was done with patch test strips using reflectance photometry with a Miditron photometer (Boehringer Mannheim-Roche, diagnostics) [14]. 
Outcome measurements

Patients who currently used oral anti-diabetics or insulin were classified as known diabetic patients. All other patients had a glucose tolerance testing (GTT) using the classic WHO criteria [15]. If the fasting blood glucose was higher than $7.8 \mathrm{mmol} / \mathrm{l}$ or 2-h GTT value was higher than $11.1 \mathrm{mmol} / \mathrm{l}$, patients were coded as de novo diabetic patients. If the fasting blood glucose was below the $7.8 \mathrm{mmol} / \mathrm{l}$ and 2-h GTT value was between 7.8-11.1 mmol/l, they were coded as impaired glucose tolerance. If the 2-h GTT value was below the $7.8 \mathrm{mmol} / \mathrm{l}$, patients were classified as normoglycaemic. Urine albumin concentration was measured in relation to the creatinin and expressed as a ratio of albumin/creatinin in $\mathrm{mg} / \mathrm{mmol}$. Microalbuminuria was defined according to the diabetic standards. Normoalbuminuria was present if the albumin-to-creatinin ratio was less than 2.5 in men and less than 3.5 in women. Microalbuminuria was present if the ratio was between 2.5 and 36 for men and between 3.5 and 40 for women. Proteinuria was defined if the ratio was above 36 for men and 40 for women. The renal function was estimated using the Cockcroft-Gault formula and normalized for BSA of $1.73 \mathrm{~m}^{2}$. The results of the urine dipstick were measured using qualitative test strips which were coded using an automated photometric reader. Leukocyturia was registered as absent, trace or positive; haematuria was registered as absent, trace or positive. During this visit, patients showed their medications. In case the patient forgot to bring the prescribed medication or medication card, the GP was contacted for the exact medication. Patients who did not use antihypertensive medication were coded as normotensive if the average blood pressure readings were below the $160 \mathrm{mmHg}$ systolic and below the $90 \mathrm{mmHg}$ diastolic. Borderline hypertensive profile was defined as diastolic blood pressure readings between 90 to $95 \mathrm{mmHg}$ and systolic blood pressure below the $160 \mathrm{mmHg}$. If patients used antihypertensive medication or had average blood pressure reading above the $160 \mathrm{mmHg}$ systolic or $95 \mathrm{mmHg}$ diastolic they were registered as hypertensive profile.

\section{Statistical analysis}

The calculations for the study size were based on a minimally detectable relative risk of 3 for microalbuminuria in relatives of Indo-Asian diabetic patients with renal failure compared with Indo-Asian diabetic patients without renal failure, with a type 1 error of 0.05 and a power of 0.90 . Based on studies in the United Kingdom [9] and the Netherlands [11], we assumed the diabetes mellitus prevalence in Indo-Asian families at 20-30\%. Assuming a prevalence of microalbuminuria in the family members of the controls at $7 \%, 150$ relatives have to be included in each family group (of whom about 40 would expect to suffer from diabetes mellitus). For statistical comparison of the difference of means, e.g. age, duration of the diabe- tes, laboratory values between the case and control group, the Student $t$ test was used; the measured difference of the means were expressed with $95 \%$ confidence intervals and $p$ values. Differences of categorical variables like glucose tolerance, urine dipstick measurements were expressed as percentage difference with $95 \%$ confidence intervals and as Chi-square $p$ values. A $p$ value of 0.05 or less was considered statistically significant.

\section{Results}

Recruitment of Index-patients. The recruitment of the index patients is shown in Table 1 . We contacted 57 index patients with Type 2 diabetes mellitus and endstage diabetic nephropathy. Of these patients 20 were not eligible: 4 could not be reached by telephone or by mail, 1 had no potential relatives for investigation, and 15 patients did not give permission to contact their relatives, leaving 37 case-index patients.

We contacted 132 control-index patients with Type 2 diabetes mellitus and no microalbuminuria according to the records of the GP's. We could not reach 26 patients because they did not respond to our invitation and could not be reached by phone. Fifteen patients had no first-degree relatives living in the Netherlands and 31 control-index patients did not give approval to contact their relatives. We therefore investigated 60 index control patients. Seventeen index control patients were excluded afterwards because they had microalbuminuria, leaving 43 eligible control-index patients for the study.

Basic characteristics of the case- and control-index patients are given in Table 2. The age at inclusion in our investigation was slightly higher in the case-index patients group than in control-index group. The caseindex patients with end-stage diabetic nephropathy also had a longer duration of diabetes disease than the control-index patients who had no nephropathy (difference 4.6 years with $95 \%$ CI 0.9 to 8.4 ). The index patients with diabetic nephropathy were more often treated with insulin therapy.

Recruitment of first-degree relatives. The recruitment of the 330 first-degree (siblings, children, parents) relatives was similar in families of index-case and indexcontrol patients . Recruitment was done in 37 case families and 43 control families. The reasons and numbers

Table 1. Recruitment and dropout reasons of the index patients

\begin{tabular}{llc}
\hline & Case-index patients & Control-index patients \\
\hline Contacted index patients & $\mathbf{5 7}$ & $\mathbf{1 3 2}$ \\
Index patients not reached & $4(7.0 \%)$ & $26(19.7 \%)$ \\
No potential family members & $1(1.8 \%)$ & $15(11.3 \%)$ \\
No Informed consent & $15(26.3 \%)$ & $31(23.5 \%)$ \\
Microalbuminuria (control-index patient) & $\mathrm{NA}$ & $17(12.9 \%)$ \\
Eligible index patients & $\mathbf{3 7}(\mathbf{6 4 . 9 \% )}$ & $\mathbf{4 3 ( 3 2 . 6 \% )}$ \\
\hline
\end{tabular}


Table 2. Basic characteristics of the eligible index patients with Type 2 diabetes mellitus

\begin{tabular}{lllr}
\hline & Case-index patients & Control-index patients & Difference (95\% CI) \\
\hline Number & 37 & 43 & $1(-20$ to 23$)$ \\
Men $n(\%)$ & $43 \%$ & $42 \%$ & $3.6(-1.2$ to 8.5$)$ \\
Age at inclusion investigation (years) & 56.1 & 52.5 & $-0.3(-5.64$ to 5.1$)$ \\
Age at diagnosis diabetes (years) & 38.7 & 39.0 & 12.6 \\
Mean diabetes duration (years) & 17.2 & 48.8 & $\mathbf{4 . 6}(\mathbf{0 . 9}$ to 8.4) \\
Insulin treated $(\%)$ & 62.2 & $\mathbf{1 3 . 4}(\mathbf{8 . 3}$ to 34.9) \\
\hline
\end{tabular}

Table 3. Basic characteristics of first-degree relatives of diabetic index patients with and without nephropathy. The characteristics are expressed as means, unless otherwise stated

\begin{tabular}{llll}
\hline & Case-relatives & Control-relatives & Difference (95\% CI) \\
\hline Age & 41.5 & 40.7 & $0.8(-1.8$ to 3.3$)$ \\
Male $(\%)$ & $37.9 \%$ & $44.7 \%$ & $-6.8(-17.5$ to 3.7$)$ \\
Body mass index $\left(\mathrm{kg} / \mathrm{m}^{2}\right)$ & 26.56 & 26.63 & $-0.07(-1.1$ to 0.9$)$ \\
Body surface area $\left(\mathrm{m}^{2}\right)$ & 1.76 & 1.80 & $-0.04(-0.1$ to 0.06$)$ \\
Waist-Hip ratio & 0.92 & 0.93 & $-0.01(-0.03$ to 0.2$)$ \\
Cholesterol & 5.2 & 5.1 & $0.1(-0.13$ to 0.3$)$ \\
HDL-Chol/cholesterol ratio & 4.21 & 4.24 & $-0.03(-0.3$ to 0.2$)$ \\
Triglycerides & 1.51 & 1.68 & $-0.17(-0.5$ to 0.1$)$ \\
HbA $(\%)$ & 5.59 & 5.75 & $-0.16(-0.5$ to 0.2$)$ \\
Glucose tolerance testing $(\mathbf{G T T})$ & & 0.89 \\
De novo DM & & $\mathbf{8 . 1 \%}$ & 0.39 \\
Impaired GT & $\mathbf{1 3 \%}$ & $\mathbf{1 0 . 6 \%}$ & $-1.7(-8.1$ to 4.7$)$ \\
Normoglycaemia & $\mathbf{8 . 9 \%}$ & $\mathbf{6 4 . 0 \%}$ & $2.9(-7.4$ to 13.2$)$ \\
Known DM & $\mathbf{6 6 . 9 \%}$ & $\mathbf{1 7 . 4 \%}$ & $-6.2(-13.7$ to 1.4$)$ \\
Age (years) & $\mathbf{1 1 . 2 \%}$ & 52.9 & $-0.1(-5.8$ to 7.6$)$ \\
Insulin usage $(\%)$ & 53.8 & 17.9 & $34.7(8.2$ to 61.3$)$ \\
Diabetes duration (years) & $\mathbf{5 2 . 6}$ & 10.0 & $0.5(-4.6$ to 5.5$)$ \\
HbA $(\%)$ & 10.5 & 8.01 & $0.25(-0.7$ to 1.2$)$ \\
\hline
\end{tabular}

of patients who did not participate in the study were distributed equally between the case and control group. In the case group 234 relatives were approached; 65 patients $(27.8 \%)$ declined or were unreachable, giving 165 case relatives for our investigation. In the control group 221 relatives were approached; 60 patients $(27.2 \%)$ declined or were unreachable, giving 161 control relatives for the present investigation.

Characteristics of the relatives. The basic characteristics are displayed in Table 3. Mean age was similar in the case and control families, about 41 years. There was a female preponderance in both family groups. Mean BMI, body surface area, waist to hip ratio measurements and lipid profiles were equal in the case and control relatives. In the case-family members, 19 $(11.2 \%)$ were known diabetic patients. The amount of known diabetic relatives in the control-family members was higher $(n=28 ; 17.4 \%)$. The results of the glucose tolerance testing according to the WHO criteria among the remaining relatives were similar.

Life style characteristics of the 330 relatives are shown in Table 4. There were no differences in numbers of smokers between the case- and control-rela- tives. There were slightly more subjects with vegetarian eating habits and Muslim religious attitudes in the control families. The level of education was distributed equally in both case- and control-relative family groups. The number of divorced persons was considerably higher than in the case group.

Blood pressure profiles and treatments are given in Table 5. The mean blood pressure measurements were equal in both the case and control relatives. Antihypertensive medical treatment was used in $13 \%$ of the case relatives and $15 \%$ of the control relatives. The distribution of the type of antihypertensive medication was not different in both groups, especially for the ACE-inhibitor and Angiotensin 2 receptor blockers usage.

Clinical features of renal disease in first-degree relatives. The features of renal disease are given in Table 6. No differences were detected in the distribution of microalbuminuria and proteinuria among the case and control relatives. Subgroup analysis for diabetic state according to the WHO criteria also showed no differences for microalbuminuria between the two family groups. Also serum creatinin values and estimated renal clearances were equal in both groups. 
Table 4. Life style in 330 first degree family relatives

\begin{tabular}{|c|c|c|c|c|}
\hline & Case relatives & Control relatives & Difference $(95 \% \mathrm{CI})$ & $p$ value \\
\hline \multicolumn{5}{|l|}{ Smoking (\%) } \\
\hline Never & 63.9 & 59.7 & \multirow{3}{*}{$4.2(-6.4$ to 14.7$)$} & \multirow[t]{3}{*}{0.32} \\
\hline Stopped & 7.1 & 11.9 & & \\
\hline Yes & 29.0 & 28.3 & & \\
\hline \multicolumn{5}{|l|}{ Vegetarian eating pattern (\%) } \\
\hline No & 95.9 & 84.3 & \multirow[t]{3}{*}{$11.6(5.2$ to 18.0$)$} & \multirow[t]{3}{*}{0.004} \\
\hline Only no meat or fish & 4.1 & 14.5 & & \\
\hline No meat, fish, dairy products or eggs & 0.0 & 1.3 & & \\
\hline \multicolumn{5}{|l|}{ Religion (\%) } \\
\hline Hindu & 75.7 & 63.5 & \multirow{4}{*}{$12.2(2.3$ to 22.1$)$} & \multirow[t]{4}{*}{$\mathbf{0 . 0 3 3}$} \\
\hline Muslim & 20.1 & 27 & & \\
\hline Christian & 1.8 & 1.9 & & \\
\hline Other or no religion & 2.4 & 7.5 & & \\
\hline \multicolumn{5}{|l|}{ Education (\%) } \\
\hline Primary school & 23.7 & 19.3 & $4.4(-4.4$ to 13.3$)$ & \multirow[t]{4}{*}{0.24} \\
\hline Lower general/vocation & 17.8 & 13.6 & $4.2(-2.4$ to 13.0$)$ & \\
\hline Intermediate and higher general/vocation & 48.4 & 54.1 & $-6.0(-16.3$ to 5.3$)$ & \\
\hline Higher vocation/University & 10.1 & 13.6 & $-2.9(-9.9$ to 3.9$)$ & \\
\hline \multicolumn{5}{|l|}{ Marital status (\%) } \\
\hline Married & 41.4 & 52.8 & $-11.4(-22.1$ to -0.6$)$ & \multirow{5}{*}{0.086} \\
\hline Unmarried & 23.7 & 25.5 & $-1.8(-11.1$ to 7.5$)$ & \\
\hline Widowed & 5.9 & 5.0 & $0.9(-3.9$ to 5.8$)$ & \\
\hline Divorced & 23.1 & 12.4 & $10.7(2.5$ to 18.8$)$ & \\
\hline Unknown & 5.9 & 4.3 & $1.6(-3.1$ to 6.3$)$ & \\
\hline
\end{tabular}

Table 5. Blood pressure and treatment of hypertension in 330 family relatives

\begin{tabular}{lcccc}
\hline & Case relatives & Control relatives & Difference (95\% CI) & $p$ value \\
\hline Systolic blood pressure (mean, mmHg) & 127.1 & 126.6 & $0.5(-4.1$ to 5.1$)$ & 0.83 \\
Diastolic blood pressure (mean, mmHg) & 79.3 & 79.7 & $-0.4(-2.84$ to 2.1$)$ & 0.75 \\
Blood pressure profile (\%) & & & \\
Normotensive profile & 73.4 & 73.3 & $0.1(-9.5$ to 9.6$)$ & 0.64 \\
Borderline hypertensive profile & 4.1 & 5.6 & $1.8(-4.8$ to 9.6$)$ & \\
Hypertensive profile & 9.5 & 6.2 & $-1.9(-9.4$ to 5.6$)$ & \\
Antihypertensive use & 13.0 & 14.9 & & \\
Anti-hypertensive medication use (\%) & & & & \\
ACE-inhibitors & 23.9 & 20.0 & $3.9(-13.6$ to 21.4$)$ & \\
AII-antagonists & 0.0 & 5.0 & $-5.0(-11.8$ to 1.7$)$ & \\
Diuretics & 26.1 & 17.5 & & \\
B-blockers & 23.9 & 20.0 & & \\
Ca- antagonists & 21.7 & 27.5 & & \\
Other & 4.4 & 10.0 & & \\
\hline
\end{tabular}

In general, there was no difference in dipstick readings between the case and control relatives.

\section{Discussion}

In this population-based family study, we found no difference in the prevalence of nephropathy in family members of Indo-Asian Type 2 diabetic patients with and without nephropathy.
Recently, we reported a close to 40 -fold higher risk of end-stage nephropathy due to Type 2 diabetes mellitus in Surinamese Indo-Asian immigrants when compared to native Dutch subjects [8] This is much higher than the eight-time higher prevalence of diabetes [11] in this population. This supports the hypothesis of a higher susceptibility to develop diabetic nephropathy in the Indo-Asian diabetic population. Another possibility is faster progression of diabetic nephropathy toward end-stage renal failure in Indo-Asian diabetic 
Table 6. Clinical features of renal disease in 330 first degree relatives of Type 2 diabetic patients with and without diabetic nephropathy

\begin{tabular}{|c|c|c|c|c|c|}
\hline & & Case relatives & Control relatives & Difference $(95 \% \mathrm{CI})$ & $p$ value \\
\hline \multicolumn{6}{|l|}{ Distribution (\%) } \\
\hline \multicolumn{2}{|l|}{ Normoalbuminuria } & 88.7 & 88.8 & $0.1(-6.7$ to 6.9$)$ & 0.81 \\
\hline \multicolumn{2}{|l|}{ Microalbuminuria } & 7.7 & 8.7 & & \\
\hline \multicolumn{2}{|l|}{ Proteinuria } & 3.6 & 2.5 & & \\
\hline \multicolumn{2}{|l|}{ Creatinin $(\mu \mathrm{mol} / \mathrm{l})$} & 83.7 & 82.7 & $1.0(-3.1$ to 5.0$)$ & 0.63 \\
\hline \multicolumn{2}{|c|}{ Cockcroft-Gault renal clearance $/ 1.73 \mathrm{~m}^{2}$} & 92.6 & 94.5 & $-1.9(-6.4$ to 2.5$)$ & 0.39 \\
\hline \multirow[t]{3}{*}{ Haematuria (\%) } & Absent & 82.8 & 80.1 & $2.7(-5.7$ to 11.1$)$ & 0.02 \\
\hline & Trace & 10.1 & 5.6 & $4.5(-1.3$ to 10.2$)$ & \\
\hline & Positive & 7.1 & 14.3 & $-7.2(-13.8$ to -0.53$)$ & \\
\hline \multirow[t]{2}{*}{ Leukocyturia (\%) } & Absent & 63.9 & 68.3 & $-4.4(-14.6$ to 5.8$)$ & 0.42 \\
\hline & Trace & 23.7 & 18.6 & $5.1(-3.7$ to 13.8$)$ & \\
\hline
\end{tabular}

patients. Earlier studies for progression of nephropathy in Indo-Asian diabetic patients were not conclusive $[16,17]$. An ethnic predisposition for renal diseases can emerge in two ways: a general susceptibility of the entire Indo-Asian population or a familial predisposition for renal diseases within certain Indo-Asian families. The latter would point towards shared environmental risk factors in these families or could indicate susceptibility genes for nephropathy which are inherited independently from diabetes mellitus.

We investigated nephropathy in first-degree relatives of Indo-Asian Type 2 diabetic patients who had end-stage diabetic nephropathy necessitating dialysis treatment or in whom preparations for dialysis were made. As controls we invited first degree relatives of Indo-Asian Type 2 diabetic patients who did not have microalbuminuria. Despite the fact that we took the most pronounced renal disease patients as case-index patients, we did not detect differences in renal disease in their relatives, defined by micro-albuminuria, glomerular filtration rates and blood pressures. The familial predisposition for Type 2 diabetes measured by GTT was similar in both the case and control relatives. There were no differences in the prevalence of newlydiscovered diabetic patients and impaired glucose tolerance test. Diabetic state, blood pressure profiles, and anti-hypertensive treatment were the same in both groups. Urine dipstick measurement for leukocyturia and haematuria were similar in the case and control relatives. A difference was noted in religions. The control group had somewhat more Muslims than Hindus which could explain the slightly higher percentage of vegetarians in the control group. However, this could only lead to less proteinuria in the control group.

The main advantage of our study is the investigation of all the diabetic family members, including previously unidentified diabetic family relatives. This appeared to be important because for every known dia- betic relative, a new diabetic relative was discovered. Diabetes was the strongest risk factor for renal disease in both family groups. To prevent bias we randomly selected the control-index patients using the records of GP's of our case-index patients. Furthermore, we invited the relatives of the case families and control families in the same way.

The findings in our study are different from other studies in other ethnic populations with Type 2 diabetic patients. In American Pima Indians, there is a higher risk of diabetic nephropathy in diabetic siblings and offspring if the index patient had diabetic nephropathy.[2] This was also found in families of Afro-American, Italian and Brazilian patients with Type 2 diabetes $[4,5,6,7]$. However, the results of these studies cannot be extrapolated directly to the Indo-Asian population living in the Netherlands. Firstly, most studies were done with relatives of known diabetic patients only as control subjects. To study the hypothesis of familial nephropathy, we also took the results of newlydiscovered diabetic family members into account. Secondly, nearly all studies used proteinuric diabetic patients as case-index patients. We investigated relatives of case-index patients on dialysis treatment, giving a stronger contrast with the relatives of control-index patients. It could be argued that the number of diabetic patients in our study was not large enough to have sufficient power for detecting a clustering of diabetic nephropathy. However, given perfect equality in the degree of albuminuria and the prevalence of nephropathy patients in both groups, it is difficult to imagine that this would dramatically change with a larger sample size.

In summary, we did not find familial clustering of renal disease in families of Type 2 diabetic patients with end-stage diabetic nephropathy. In an earlier study the much higher incidence of diabetic nephropathy in Indo-Asians is not simply due to the higher incidence of diabetes. The "gap" between the 40-fold 
increase in diabetic nephropathy and the only 8-fold increase of diabetes itself may mean that all persons of Indo-Asian descent are especially vulnerable to develop nephropathy once they have developed diabetes mellitus. Another possibility is faster progression of nephropathy until end-stage diabetic nephropathy in this population.

Acknowledgements. We would like to express our gratitude to the Dutch Diabetes Research Foundation for supporting our study. We thank Mrs. J. Krol for her valuable and indispensable assistance in this project. In addition we thank Mrs. M. van Aartrijk, Mr. F. Baboe and Mrs. A. Doerga for their practical assistance. We thank Dr. M. Frölich for the laboratory procedures and measurements. We are grateful for the collaboration with the nephrologists, internists and general practitioners in the city of The Hague. We appreciate the secretarial support by Mrs. I. Abelman and Mrs.T. van der Ham.

\section{References}

1. Seaquist ER, Goetz FC, Rich S, Barbosa J (1989) Familial clustering of diabetic kidney disease. Evidence for genetic susceptibility to diabetic nephropathy. N Engl J Med 320:1161-1165

2. Pettitt DJ, Saad MF, Bennett PH, Nelson RG, Knowler WC (1990) Familial predisposition to renal disease in two generations of Pima Indians with type 2 (non-insulin-dependent) diabetes mellitus. Diabetologia 33:438443

3. Imperatore G, Hanson RL, Pettitt DJ, Kobes S, Bennett PH, Knowler WC (1998) Sib-pair linkage analysis for susceptibility genes for microvascular complications among Pima Indians with type 2 diabetes. Pima Diabetes Genes Group. Diabetes 47:821-830

4. Freedman BI, Tuttle AB, Spray BJ (1995) Familial predisposition to nephropathy in African-Americans with noninsulin-dependent diabetes mellitus. Am J Kidney Dis 25:710-713

5. Canani LH, Gerchman F, Gross JL (1999) Familial clustering of diabetic nephropathy in Brazilian type 2 diabetic patients. Diabetes 48:909-913
6. Faronato PP, Maioli M, Tonolo G et al. (1997) Clustering of albumin excretion rate abnormalities in Caucasian patients with NIDDM. The Italian NIDDM Nephropathy Study Group. Diabetologia 40:816-823

7. Fava S, Azzopardi J, Hattersley AT, Watkins PJ (2000) Increased prevalence of proteinuria in diabetic sibs of proteinuric type 2 diabetic subjects. Am J Kidney Dis 35:708-712

8. Chandie Shaw PK, Vandenbroucke JP, Tjandra YI et al. (2002) Increased end-stage diabetic nephropathy in IndoAsian immigrants living in the Netherlands. Diabetologia 45:337-341

9. Burden AC, McNally PG, Feehally J, Walls J (1992) Increased incidence of end-stage renal failure secondary to diabetes mellitus in Asian ethnic groups in the United Kingdom. Diabet Med 9:641-645

10. Lightstone L, Rees AJ, Tomson C, Walls J, Winearls CG, Feehally J (1995) High incidence of end-stage renal disease in Indo-Asians in the UK. QJM 88:191-195

11. Middelkoop BJC, Kesarlal-Sadhoeram SM, Ramsaransing GN, Struben HWA (1999) Diabetes mellitus among South Asian inhabitants of the Hague: high prevalence and an age-specific socioeconomic gradient. Int $\mathrm{J}$ Epidemiol 28:1119-1123

12. Vijay V, Snehalatha C, Shina K, Lalitha S, Ramachandran A (1999) Familial aggregation of diabetic kidney disease in Type 2 diabetes in south India. Diabetes Res Clin Pract 43:167-171

13. Cockcroft DW, Gault MH (1976) Prediction of creatinine clearance from serum creatinine. Nephron 16:31-41

14. Marx A.M., Kropf M.J., Gressner A.M. (1989) On the performance an reliability of mechanized urine teststrip measurement in comparison with visual reading. J Clin Chem Clin Biochem 27:433-443

15. Expert Committee on the Diagnosis and Classification of Diabetes Mellitus (1997) Report of the Expert Committee on the Diagnosis and Classification of Diabetes Mellitus. Diabetes Care 20:1183-1197

16. Koppiker N, Feehally J, Raymond N, Abrams KR, Burden AC (1998) Rate of decline in renal function in Indo-Asians and Whites with diabetic nephropathy. Diabet Med 15:60-65

17. Earle KK, Porter KA, Ostberg J, Yudkin JS (2001) Variation in the progression of diabetic nephropathy according to racial origin. Nephrol Dial Transplant 16:286-290 